\title{
Complete-active-space multiconfiguration Dirac-Hartree-Fock calculations of hyperfine-structure constants of the gold atom
}

\author{
Jacek Bieroń \\ Instytut Fizyki imienia Mariana Smoluchowskiego, Uniwersytet Jagielloński, Reymonta 4, 30-059 Kraków, Poland \\ Charlotte Froese Fischer \\ National Institute of Standards and Technology, Gaithersburg, Maryland 20899-8420, USA \\ Paul Indelicato \\ Laboratoire Kastler Brossel, École Normale Supérieure, CNRS, Université Pierre et Marie Curie-Paris 6, \\ Case 74, 4 Place Jussieu, 75252 Paris CEDEX 05, France \\ Per Jönsson \\ Nature, Environment, Society, Malmö University, S-205 06 Malmö, Sweden \\ Pekka Pyykkö \\ Department of Chemistry, University of Helsinki, P.O. Box 55, A.I. Virtasen Aukio 1, 00014 Helsinki, Finland \\ (Received 28 February 2009; published 4 May 2009)
}

\begin{abstract}
The multiconfiguration Dirac-Hartree-Fock model has been employed to calculate the expectation values for the hyperfine splittings of the $5 d^{9} 6 s^{2}{ }^{2} D_{3 / 2}$ and $5 d^{9} 6 s^{2}{ }^{2} D_{5 / 2}$ levels of atomic gold. One-, two-, and three-body electron correlation effects involving all 79 electrons have been included in a systematic manner. The approximation employed in this study is equivalent to a complete-active-space approach. Calculated electric field gradients, together with experimental values of the electric quadrupole hyperfine-structure constants, allow us to extract a nuclear electric quadrupole moment $Q\left({ }^{197} \mathrm{Au}\right)=521.5(5.0) \mathrm{mb}$.
\end{abstract}

DOI: $10.1103 /$ PhysRevA.79.052502

PACS number(s): 31.15.am, 31.15.vj, 31.30.Gs, 21.10.Ky

\section{INTRODUCTION}

$A b$ initio calculations of atomic properties can now be performed routinely both in the framework of the multiconfiguration Dirac-Hartree-Fock (MCDHF) theory [1-5], as well as many-body perturbation theory (MBPT) [6-9]. Both these methods are designed to evaluate in a systematic manner the electron-electron correlation effects, which constitute the dominant correction to all $a b$ initio calculations based on the central-field approach. However, the complexity increases rapidly with the atomic number, and fully correlated calculations, in which all electrons are explicitly correlated, are still possible only for very light elements (see, e.g., [10-12] for model calculations of hyperfine constants of lithiumlike systems). For heavy atoms both theories can only be applied in a limited model (one- and two-body correlation effects) or only to certain atoms (closed-shell systems or alkali-metal-like systems). The main purpose of the present paper was to carry out an accurate calculation of hyperfinestructure constants of a heavy atom within the framework of the MCDHF theory. The calculations described in the present paper constitute a successful evaluation of one-, two-, and three-body electron correlation effects for a heavy, openshell, neutral atom. The multiconfiguration model applied in the present paper is effectively equivalent to a completeactive-space (CAS) approach in the sense that in the calculation of the hyperfine electric quadrupole moments all nonnegligible electron correlation effects were explicitly accounted for at a $1 \%$ level of precision or better. The gold atom has been chosen because the hyperfine structures [13-16], the nuclear electric quadrupole moments [17-21], and other properties [22-24] of gold have been the subject of much activity recently (the latest summary of nuclear quadrupole moments is given in Ref. [25]). The second objective of the present paper is to evaluate the electric quadrupole moment $Q$ of the ${ }^{197} \mathrm{Au}$ isotope.

\section{THEORY}

The numerical-grid wave functions [1] were generated as the self-consistent solutions of the Dirac-Hartree-Fock equations [26] in systematically increasing multiconfiguration bases (of size NCF, which is a commonly used shorthand of "number of configuration functions") of symmetry-adapted configuration state functions (CSFs) $\Phi\left(\gamma_{k} J\right)$,

$$
\Psi(J)=\sum_{k}^{\mathrm{NCF}} c_{k} \Phi\left(\gamma_{k} J\right),
$$

where $\Psi(J)$ is an eigenfunction of even parity and of total angular momentum $J$ for each of the two states $\Psi\left(5 d^{9} 6 s^{2}{ }^{2} D_{3 / 2}\right)$ and $\Psi\left(5 d^{9} 6 s^{2}{ }^{2} D_{5 / 2}\right)$ of the isotope ${ }_{79}^{197} \mathrm{Au}$. The sets $\gamma_{k}$ describe multiconfiguration expansions, for which configuration mixing coefficients $c_{k}$ were obtained through diagonalization of the Dirac-Coulomb Hamiltonian,

$$
H_{D C}=\sum_{i}\left[c \boldsymbol{\alpha}_{i} \cdot \boldsymbol{p}_{i}+\left(\beta_{i}-1\right) c^{2}+V\left(r_{i}\right)\right]+\sum_{i>j} 1 / r_{i j} .
$$

All calculations were done with the nucleus modeled as a sphere, where a two-parameter Fermi distribution [27] was employed to approximate the radial dependence of the 
nuclear charge density. The nuclear magnetic dipole moment $\mu=0.145746(9) \mu_{N}$ of ${ }_{79}^{197} \mathrm{Au}$ has been used in calculations of magnetic dipole hyperfine constants [28,29].

\section{METHOD}

The numerical wave functions were obtained independently for the two levels of interest, $5 d^{9} 6 s^{2}{ }^{2} D_{3 / 2}$ and $5 d^{9} 6 s^{2}{ }^{2} D_{5 / 2}$. The calculations proceeded in eight phases:

(1) Spectroscopic orbitals were obtained in the DiracHartree-Fock approximation. These were kept frozen in all subsequent calculations.

(2) Virtual orbitals were generated in an approximation (called SrD and explained in Sec. III A), in which all single and restricted double substitutions from 3 spd $4 s p d f 5 s p d 6 s$ spectroscopic orbitals to eight layers of virtual orbitals were included (see Sec. III A for definitions of spectroscopic and virtual orbital sets).

(3) Contributions from $1 s 2 s p$ shells were added in the configuration-interaction (CI) calculation, i.e., with all orbitals frozen. Only single substitutions contributed to the expectation values. The configurations involving $1 s 2 s p$ orbitals were carried over to the following phases.

(4) Unrestricted single and double substitutions (SD) were performed, in which one or two occupied orbitals from the $5 s p d 6 s$ subshells were replaced by orbitals from the virtual set " $3 s p d f 2 g 1 h$," i.e., three virtual orbitals of each of the " $s$," " $p$, , " $d$," and " $f$ " symmetries, plus two virtual orbitals of the " $g$ " symmetry, and one virtual orbital of the " $h$ " symmetry.

(5) Unrestricted triple substitutions (T) from 5 spd6s valence and core orbitals to " $2 s p d f 1 g$ " virtual set were added.

(6) The final series of configuration-interaction calculations were based on the multiconfiguration expansions carried over and merged from all previous phases enumerated above.

(7) Contributions from the Breit interaction were evaluated in the single-configuration approximation, including the full Breit operator in the self-consistent-field process.

(8) The values of the nuclear electric quadrupole moment $Q\left({ }^{197} \mathrm{Au}\right)$ were obtained from the relation $B(J)$ $=2 e Q\left\langle J J\left|T^{(2)}\right| J J\right\rangle$, where the electronic operator $T^{(2)}$ represents the electric field gradient at the nucleus. Expectation values of hyperfine constants $A$ and of electric field gradients were calculated [30] separately for both states, ${ }^{2} D_{3 / 2}$ and ${ }^{2} D_{5 / 2}$. The experimental values of the hyperfine constants $A$ and $B$ were taken from $[31,32]$.

\section{A. Virtual orbital set}

We generated eight layers of virtual shells (three layers with "spdfgh" symmetries and five layers with "spdfg" symmetries). It should be noted that the notion of a "layer" is somewhat different when applied to occupied (also referred to as spectroscopic) orbitals, as opposed to virtual (also referred to as correlation) orbitals. A core layer, i.e., a subset of occupied orbitals possessing the same principal quantum number (often referred to as a shell), constitutes a set of one-electron spin orbitals, clustered in space and having similar one-electron energy values. On the other hand, virtual
TABLE I. Calculated values of $A$ and $Q$ obtained in several approximations during the process of generation of virtual orbital set for the $D_{3 / 2}$ state. DHF-uncorrelated Dirac-Hartree-Fock value; $n$-largest principal quantum number in the orbital set; from-spectroscopic orbitals opened for $\mathrm{SrD}$ substitutions; tovirtual orbital set; and $\mathrm{NCF}$ - number of configurations (see text for further details).

\begin{tabular}{ccccccc}
\hline \hline \multicolumn{9}{c}{ Expt. } & & & \multicolumn{2}{c}{$199.8425(2)$} \\
\cline { 1 - 3 } \cline { 6 - 7 }$n$ & from & to & NCF & & $\begin{array}{c}A \\
(\mathrm{MHz})\end{array}$ & $\begin{array}{c}Q \\
(\mathrm{mb})\end{array}$ \\
\hline DHF & & & 1 & & 218.011 & 580.807 \\
7 & $5 d 6 s$ & $1 s p d f g h$ & 1147 & & 187.302 & 623.275 \\
8 & $5 s p d 6 s$ & $2 s p d f g h$ & 13729 & & 198.774 & 652.057 \\
9 & $4 s p d f \cdots 6 s$ & $3 s p d f g h$ & 97526 & & 195.492 & 547.891 \\
10 & $3 s p d \cdots 6 s$ & $4 s p d f g 3 h$ & 222129 & & 196.513 & 528.752 \\
11 & $3 s p d \cdots 6 s$ & $5 s p d f g 3 h$ & 222494 & & 199.413 & 523.736 \\
12 & $3 s p d \cdots 6 s$ & $6 s p d f g 3 h$ & 222851 & & 199.455 & 514.186 \\
13 & $3 s p d \cdots 6 s$ & $7 s p d f g 3 h$ & 223212 & & 200.431 & 515.489 \\
14 & $3 s p d \cdots 6 s$ & $8 s p d f g 3 h$ & 223573 & & 199.871 & 515.495 \\
\hline \hline
\end{tabular}

orbitals with the same principal quantum number are not necessarily spatially clustered because their one-electron energy values do not have physical meaning and may vary widely, depending on the correlation effects that a particular virtual orbital describes. Therefore a "virtual layer" usually means a subset of the virtual set, generated in one step of the procedure, as described below. Such a layer is often composed of orbitals with different angular symmetries. The notation used in the tables and text of the present paper reflects the above considerations in the sense that occupied orbitals are listed by their principal and angular quantum numbers (i.e., $5 s p d$ means three occupied orbitals of $s, p$, and $d$ symmetries with principal quantum number $n=5$ ), while virtual orbitals are listed by angular symmetry and quantity (i.e., " 5 spd" would mean 15 virtual orbitals-five of each of the "s," " $p$," and " $d$ " symmetries). To avoid confusion we distinguish occupied orbitals from virtual ones in the present paper by using italics for occupied orbitals, while virtual orbitals are enclosed in quotation marks. This distinction is not applied in the tables since in the tables there are always headings "from" and "to" which clearly denote occupied and virtual orbitals, respectively. The notation should always be analyzed in the proper context (see [33] for further details). In the present calculations single and restricted double (SrD) substitutions were allowed from valence and core orbitals (starting from $5 d 6 s$ for the first virtual layer). The restriction was applied to double substitutions in such a way that only one electron was substituted from core 3 spd4spdf5spd shells, the other one had to be substituted from valence $6 \mathrm{~s}$ shell. Each subsequent layer was generated with substitutions from deeper core shells down to $3 \mathrm{~s}$. Table I shows which occupied orbitals were opened at each step, as well as composition of the virtual orbital set when subsequent layers were generated for the $5 d^{9} 6 s^{2}{ }^{2} D_{3 / 2}$ state. For instance, the line marked " 10 " in the first column describes the generation of the fourth virtual layer, for which the largest principal 
TABLE II. Calculated values of $A$ and $Q$ obtained in several approximations during the process of generation of virtual orbital set for the $D_{5 / 2}$ state. DHF-uncorrelated Dirac-Hartree-Fock value; $n$-largest principal quantum number in the orbital set; from-spectroscopic orbitals opened for $\mathrm{SrD}$ substitutions; tovirtual orbital set; and $\mathrm{NCF}$ - number of configurations (see text for further details).

\begin{tabular}{|c|c|c|c|c|c|}
\hline \multicolumn{4}{|c|}{ Expt. } & \multicolumn{2}{|c|}{$80.236(3)$} \\
\hline$n$ & from & to & $\mathrm{NCF}$ & $\begin{array}{c}A \\
(\mathrm{MHz})\end{array}$ & $\underset{(\mathrm{mb})}{Q}$ \\
\hline DHF & & & 1 & 79.041 & 612.985 \\
\hline 7 & $5 d 6 s$ & $1 s p d f g h$ & 11984 & 69.487 & 707.216 \\
\hline 8 & $5 s p d 6 s$ & $2 s p d f g h$ & 33291 & 72.278 & 673.387 \\
\hline 9 & $4 s p d f \cdots 6 s$ & $3 s p d f g h$ & 128639 & 77.761 & 558.526 \\
\hline 10 & $3 s p d \cdots 6 s$ & $4 s p d f g 3 h$ & 290612 & 81.020 & 532.862 \\
\hline 11 & $3 s p d \cdots 6 s$ & $5 s p d f g 3 h$ & 291039 & 81.045 & 534.635 \\
\hline 12 & $3 s p d \cdots 6 s$ & $6 s p d f g 3 h$ & 291466 & 81.248 & 520.409 \\
\hline 13 & $3 s p d \cdot \cdots 6 s$ & $7 s p d f g 3 h$ & 291893 & 81.214 & 520.890 \\
\hline 14 & $3 s p d \cdots 6 s$ & $8 s p d f g 3 h$ & 292320 & 82.136 & 520.259 \\
\hline
\end{tabular}

quantum number was 10. All occupied orbitals between $3 s$ and $6 s$ (i.e., $3 s p d 4 s p d f 5 s p d 6 s$ ) were opened for substitutions; the virtual set was composed of four orbitals of symmetries " $s, "$ " $p, "$ " $d$, , " $f$," " $g$," and three orbitals of " $h$ " symmetry.

The last four layers (those with principal quantum numbers 11-14) were generated with a further restriction, which allowed only single substitutions to these last layers.

Table II presents the analogous data obtained in the process of generation of virtual orbital layers for the $5 d^{9} 6 s^{2}{ }^{2} D_{5 / 2}$ state. The data from both tables are also presented as red lines with no symbols in Fig. 3.

\section{B. Contributions from $1 s 2 s p$ orbitals}

After generating the virtual orbital set, all orbitals were frozen and further calculations were carried out in the CI approach. First, the effects of $1 s 2 s p$ orbitals were evaluated in separate CI calculations. For the $5 d^{9} 6 s^{2}{ }^{2} D_{3 / 2}$ state they are presented in Table III, together with the contributions of all other occupied orbitals of the gold atom. The orbitals that were open for single and restricted double substitutions to the full virtual set are listed in the first column. The contributions of individual orbitals (i.e., of the leftmost orbital in the first column) are listed in the fourth and sixth columns and presented in graphical form in Fig. 1. The individual contributions of the $2 p, 2 s$, and $1 s$ orbitals to the total $Q$ value were on the order of $0.6 \%, 0.2 \%$, and $0.02 \%$, respectively. The combined contribution of $1 s 2 s p$ shells was on the order of $0.8 \%$, with respect to the total $Q$ value. The contribution to the calculated value of magnetic dipole hyperfine constant $A$ was evaluated in the same manner as for $Q$.

A similar procedure has been carried out for the $Q$ and $A$ values of the $5 d^{9} 6 s^{2}{ }^{2} D_{5 / 2}$ state. The results for the ${ }^{2} D_{5 / 2}$ state are shown in Table IV and in Fig. 2. The individual
TABLE III. Contributions from occupied orbitals to the calculated values of $A$ and $Q$ for the $5 d^{9} 6 s^{2}{ }^{2} D_{3 / 2}$ state of Au; orbitals $=$ set of orbitals open for single and restricted double substitutions from all shells listed in the first column to the full virtual set; $\mathrm{NCF}=$ size of the multiconfiguration expansion; $\Delta A=$ contribution $(\mathrm{MHz})$ of the leftmost orbital from a given orbital set to the total $A$ value (i.e., the individual contribution of the $1 s$ orbital is listed in the line $1 s \ldots 6 s)$; and $\Delta Q=$ contribution $(\mathrm{mb})$ of the leftmost orbital from a given set to the $Q$ value.

\begin{tabular}{|c|c|c|c|c|c|}
\hline orbitals & $\mathrm{NCF}$ & $\begin{array}{c}A \\
(\mathrm{MHz})\end{array}$ & $\Delta A$ & $\underset{(\mathrm{mb})}{Q}$ & $\Delta Q$ \\
\hline & 1 & 218.011 & & 580.807 & \\
\hline $5 d 6 s$ & 16457 & 189.406 & -28.605 & 613.418 & 32.611 \\
\hline $5 p d 6 s$ & 39808 & 153.103 & -36.302 & 629.418 & 16.000 \\
\hline $5 s p d 6 s$ & 48129 & 190.849 & 37.746 & 625.559 & -3.859 \\
\hline $4 f 5 s p d 6 s$ & 89477 & 187.661 & -3.188 & 623.738 & -1.821 \\
\hline $4 d f 5 s p d 6 s$ & 124673 & 194.614 & 6.953 & 593.938 & -29.800 \\
\hline $4 p d f 5 s p d 6 s$ & 148188 & 202.721 & 8.107 & 528.442 & -65.496 \\
\hline $4 s p d f 5 s p d 6 s$ & 156525 & 196.476 & -6.245 & 529.646 & 1.204 \\
\hline $3 d 4 s p d f 5 s p d 6 s$ & 191721 & 199.346 & 2.870 & 525.342 & -4.304 \\
\hline $3 p d \cdots 6 s$ & 215236 & 201.106 & 1.760 & 514.175 & -11.167 \\
\hline $3 s p d \cdots 6 s$ & 223573 & 199.872 & -1.234 & 515.495 & 1.320 \\
\hline $2 p 3 s p d \cdots 6 s$ & 247088 & 196.564 & -3.308 & 518.635 & 3.140 \\
\hline $2 s p 3 s p d \cdots 6 s$ & 255425 & 199.576 & 3.012 & 519.539 & 0.904 \\
\hline $1 s 2 s p 3 s p d \cdots 6 s$ & 263762 & 199.554 & -0.022 & 519.634 & 0.095 \\
\hline
\end{tabular}

contributions of the $2 p, 2 s$, and $1 s$ orbitals to the total $Q$ value were on the order of $0.5 \%, 0.2 \%$, and $0.02 \%$, respectively. The combined contribution of $1 s 2 s p$ shells was on the order of $0.7 \%$, with respect to the total $Q$ value.

All these contributions have been included in the $Q$ and $A$ values obtained within the $\mathrm{SrD}$ approximation and the CSFs involved in evaluation of these contributions were carried over to all subsequent calculations.

It should be pointed out that the data in Tables III and IV and in Figs. 1 and 2 were obtained with single and restricted double substitutions, i.e., with unrestricted double and triple substitutions excluded. Therefore the contributions of the 5psd and 4spdf shells are somewhat distorted-if double

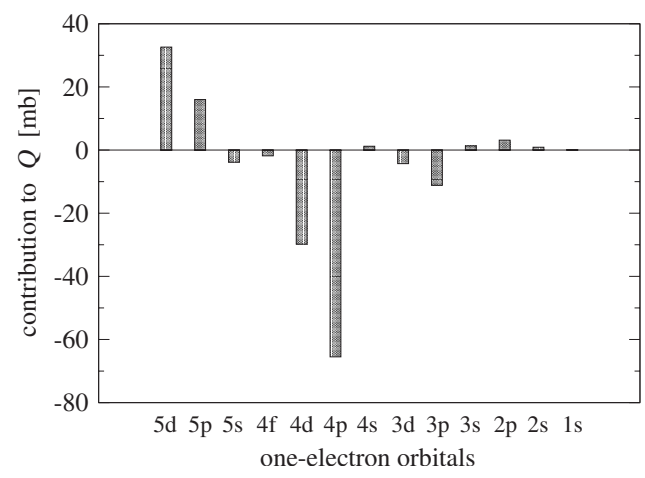

FIG. 1. Contributions from occupied orbitals to the calculated value of $Q$ for the $5 d^{9} 6 s^{2}{ }^{2} D_{3 / 2}$ state of Au. See caption of Table III and Sec. III B for further details. 
TABLE IV. Contributions from occupied orbitals to the calculated values of $A$ and $Q$ for the $5 d^{9} 6 s^{2}{ }^{2} D_{5 / 2}$ state of $\mathrm{Au}$; orbitals $=$ set of orbitals open for single and restricted double substitutions from all shells listed in the first column to the full virtual set; $\mathrm{NCF}=$ size of the multiconfiguration expansion; $\Delta A=$ contribution $(\mathrm{MHz})$ of the leftmost orbital from a given orbital set to the total $A$ value (i.e., the individual contribution of the $1 s$ orbital is listed in the line $1 s \ldots 6 s) ; \Delta Q=$ contribution $(\mathrm{mb})$ of the leftmost orbital from a given set to the $Q$ value.

\begin{tabular}{|c|c|c|c|c|c|}
\hline orbitals & $\mathrm{NCF}$ & $\begin{array}{c}A \\
(\mathrm{MHz})\end{array}$ & $\Delta A$ & $\underset{(\mathrm{mb})}{Q}$ & $\Delta Q$ \\
\hline & 1 & 79.041 & & 612.985 & \\
\hline $5 d 6 s$ & 21501 & 106.724 & 27.683 & 651.547 & 38.562 \\
\hline $5 p d 6 s$ & 51800 & 109.554 & 2.830 & 643.451 & -8.096 \\
\hline $5 s p d 6 s$ & 62536 & 71.472 & -38.082 & 638.694 & -4.757 \\
\hline $4 f 5 s p d 6 s$ & 117626 & 70.636 & -0.836 & 636.280 & -2.414 \\
\hline $4 d f 5 s p d 6 s$ & 163739 & 73.490 & 2.854 & 604.280 & -32.000 \\
\hline $4 p d f 5 s p d 6 s$ & 194221 & 74.597 & 1.107 & 532.632 & -71.648 \\
\hline $4 s p d f 5 s p d 6 s$ & 204973 & 79.767 & 5.170 & 534.008 & 1.376 \\
\hline $3 d 4 s p d f 5 s p d 6 s$ & 251086 & 80.877 & 1.110 & 530.308 & -3.700 \\
\hline $3 p d \cdot \cdots 6 s$ & 281568 & 80.580 & -0.297 & 518.734 & -11.574 \\
\hline $3 s p d \cdots 6 s$ & 292320 & 82.136 & 1.556 & 520.259 & 1.525 \\
\hline $2 p 3 s p d \cdots 6 s$ & 322802 & 81.700 & -0.436 & 522.677 & 2.418 \\
\hline $2 s p 3 s p d \cdots 6 s$ & 333554 & 78.995 & -2.705 & 523.757 & 1.080 \\
\hline $1 s 2 s p 3 s p d \cdots 6 s$ & 344306 & 79.025 & 0.030 & 523.880 & 0.123 \\
\hline
\end{tabular}

and triple substitutions were included, the individual contributions of the $5 p s d$ and $4 s p d f$ shells would differ by a few percent. Only the $3 s p d, 2 s p$, and $1 s$ shells are essentially insensitive to double and triple substitutions (see Sec. III C below). Therefore their contributions are approximately correct.

\section{Double, triple, and quadruple substitutions}

The decomposition of the electron correlation correction to the hyperfine structure into one-, two-, three-, and fourbody effects can be understood from the following (simplified) analysis. The structure of the $5 d^{9} 6 s^{22} D$ states of gold is

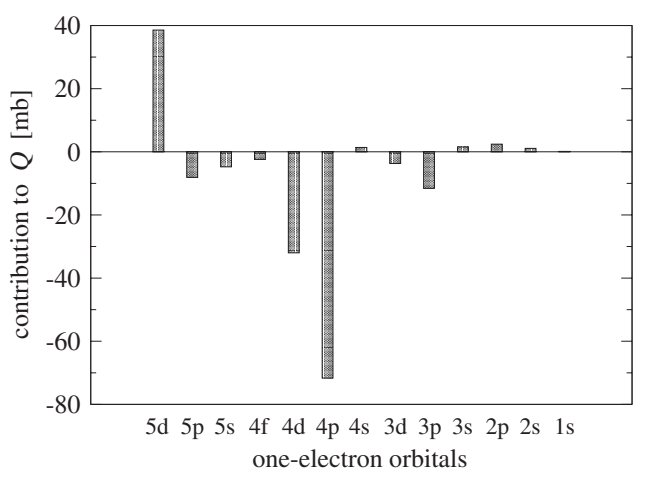

FIG. 2. Contributions from occupied orbitals to the calculated value of $Q$ for the $5 d^{9} 6 s^{2}{ }^{2} D_{5 / 2}$ state of Au. See caption of Table IV and Sec. III B for further details.
TABLE V. Values of $Q$ and $A$ for the $D_{3 / 2}$ state, calculated in configuration-interaction approach, with single and unrestricted double substitutions, in several different multiconfiguration expansions; from-spectroscopic orbitals opened for substitutions; tovirtual orbital set; and $\mathrm{NCF}$ - number of configurations (see text for further details).

\begin{tabular}{|c|c|c|c|c|}
\hline \multicolumn{3}{|c|}{ Expt. } & \multicolumn{2}{|c|}{$199.8425(2)$} \\
\hline from & to & $\mathrm{NCF}$ & $\begin{array}{c}A \\
(\mathrm{MHz})\end{array}$ & $\underset{(\mathrm{mb})}{Q}$ \\
\hline $5 s p d 6 s$ & $1 s p d f g h$ & 259135 & 205.426 & 521.191 \\
\hline $4 s p d f 5 s p d 6 s$ & $1 s p d f g h$ & 358019 & 205.968 & 521.503 \\
\hline $5 s p d 6 s$ & $2 s p d f$ & 279559 & 210.523 & 509.839 \\
\hline $5 s p d 6 s$ & $2 s p d f g$ & 320545 & 211.512 & 511.461 \\
\hline $5 s p d 6 s$ & $2 s p d f g h$ & 366257 & 211.480 & 512.286 \\
\hline $5 s p d 6 s$ & $3 s p d f 2 g 1 h$ & 459594 & 213.088 & 510.451 \\
\hline $5 s p d 6 s$ & $3 s p d f 2 g h$ & 465794 & 213.075 & 510.402 \\
\hline $5 s p d 6 s$ & $3 s p d f g 2 h$ & 506987 & 213.146 & 510.268 \\
\hline $5 s p d 6 s$ & $4 s p d f 2 g h$ & 687301 & 213.200 & 510.478 \\
\hline
\end{tabular}

determined to a large extent by the interaction of the valence $6 s^{2}$ shell with a highly polarizable $5 d^{9}$ shell. The direct and indirect effects of relativity bring the outer $d$ shell much closer, radially and energetically, to the valence $s$ orbital than in homologous silver and copper atoms [22,34]. This in turn increases the polarization of the $5 d^{9}$ shell by the valence electrons. Therefore, the core-valence interaction (the leading electron correlation correction) leads to the contraction of the $6 s$ orbital, which overestimates the hyperfine structure. The unrestricted double substitutions affect the hyperfine structure in two ways: directly through the CSFs themselves but also indirectly through the change in the expansion coefficients of the important configurations obtained by single substitutions. Three-particle effects in turn affect the expansion coefficients of the configurations obtained from double substitutions. In a simple picture we can describe the wave function in terms of pair-correlation functions and the threeparticle effects then account for polarization of paircorrelation functions, leading to an increase in the hyperfine structure [35]. Four-particle effects affect mostly the expansion coefficients of the configurations obtained from double substitutions. Therefore their influence on the hyperfine structure is indirect and second order to that of the double substitutions. They are usually small and can often be neglected [36]; they are discussed in Sec. III C.

Tables V and VI show the results of configurationinteraction calculations, where various combinations of occupied and virtual sets were tested with single and unrestricted double substitutions. The data from both tables are also presented as empty circles in Fig. 3.

The second line in Tables V and VI represents a calculation in which substitutions from the $4 s p d f$ shells were allowed to one layer of virtual orbitals. When compared with the first line, it yields the effect of $4 s p d f$ shells on the calculated values of $Q$ and $A$. In order to limit the size of the configuration expansions, the CSFs representing the above substitutions were not carried over to the following higher- 
TABLE VI. Values of $Q$ and $A$ for the $D_{5 / 2}$ state, calculated in configuration-interaction approach, with single and unrestricted double substitutions, in several different multiconfiguration expansions; from-spectroscopic orbitals opened for substitutions; tovirtual orbital set; and $\mathrm{NCF}$ - number of configurations (see text for further details).

\begin{tabular}{ccccccc}
\hline \hline \multicolumn{3}{c}{ Expt. } & & & \multicolumn{2}{c}{$80.236(3)$} \\
\cline { 6 - 7 } \cline { 5 - 6 } from & to & NCF & & $\begin{array}{c}A \\
(\mathrm{MHz})\end{array}$ & $\begin{array}{c}Q \\
(\mathrm{mb})\end{array}$ \\
\hline 5spd6s & 1 1spdfgh & 339306 & & 74.258 & 507.823 \\
4spdf5spd6s & 1 1spdfgh & 467381 & & 72.048 & 509.321 \\
$5 s p d 6 s$ & $2 s p d f g h$ & 480824 & & 73.468 & 512.278 \\
$5 s p d 6 s$ & $3 s p d f 2 g h$ & 607421 & & 73.494 & 512.559 \\
$5 s p d 6 s$ & $4 s p d f 3 g h$ & 898368 & & 73.294 & 514.621 \\
$5 s p d 6 s$ & $5 s p d f 4 g 3 h$ & 1228675 & 73.212 & 514.269 \\
\hline \hline
\end{tabular}

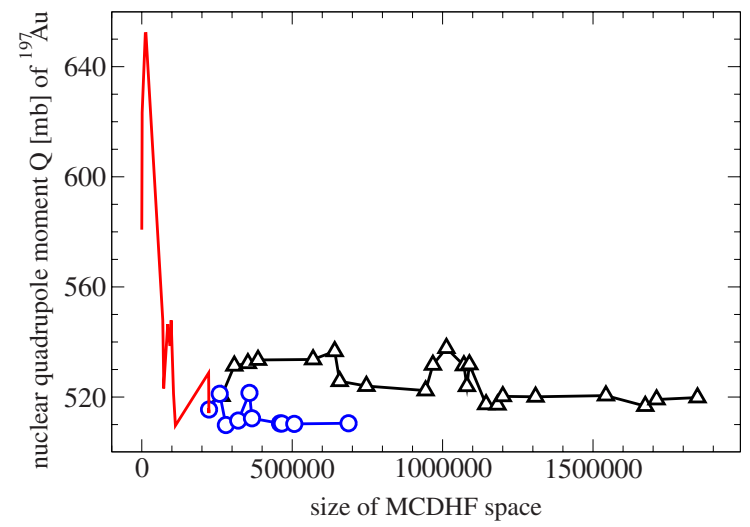

(a) from electric field gradient and

$$
\mathrm{B}\left(5 d^{9} 6 s^{2}{ }^{2} D_{3 / 2}\right)
$$

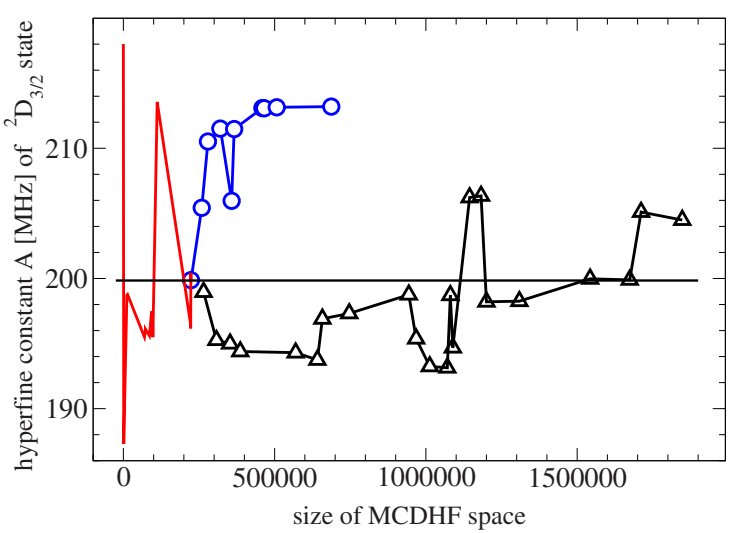

(c) $\mathrm{A}\left(5 d^{9} 6 s^{2}{ }^{2} D_{3 / 2}\right)$ order calculations. Instead, the corrections were included additively, as described in Sec. IV A. At the same time the evaluation of these corrections may be treated as a crude estimate of error arising from omitted double substitutions from occupied shells (see Sec. IV B for details).

An inspection of the last column of Table $\mathrm{V}$ indicates that three layers of virtual orbitals were necessary to reach convergence of the $Q$ and $A$ values in the single and unrestricted double substitutions (SD) approximation for the $5 d^{9} 6 s^{2}{ }^{2} D_{3 / 2}$ state. Four layers were necessary in case of the $5 d^{9} 6 s^{2}{ }^{2} D_{5 / 2}$ state (see Table VI).

Tables VII and VIII show the results of configurationinteraction calculations, in which various combinations of occupied and virtual sets were tested with unrestricted double and triple substitutions. The data from both tables are also presented as triangles in Fig. 3. Two layers of virtual orbitals were necessary to reach convergence of the $Q$ value in the single, double, and triple substitution (SDT) approxi-

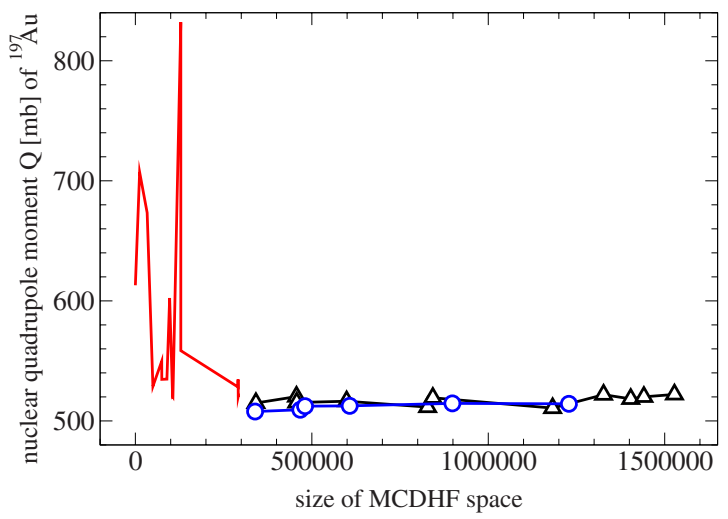

(b) from electric field gradient and

$$
\mathrm{B}\left(5 d^{9} 6 s^{2}{ }^{2} D_{5 / 2}\right)
$$

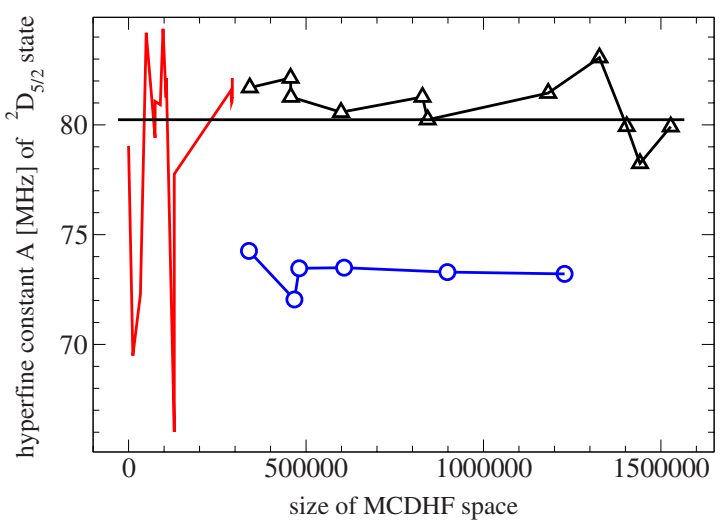

(d) $\mathrm{A}\left(5 d^{9} 6 s^{2}{ }^{2} D_{5 / 2}\right)$

FIG. 3. (Color online) Nuclear quadrupole moment $Q\left({ }^{197} \mathrm{Au}\right)(\mathrm{mb})$ obtained from the calculated electric field gradients, and hyperfine magnetic dipole constants $A(\mathrm{MHz})$ of the states $5 d^{9} 6 s^{2}{ }^{2} D_{3 / 2}$ and $5 d^{9} 6 s^{2}{ }^{2} D_{5 / 2}$, as functions of the size of the multiconfiguration expansions; line with no symbols (red online)—SrD approximation; circles (blue online)—SD approximation; triangles (black online) — SDT and final CAS approximations (see text for details). Horizontal straight lines in (c) and (d) represent the experimental values of hyperfine constants, $A\left({ }^{2} D_{3 / 2}\right)=199.8425(2) \mathrm{MHz}[31]$ and $A\left({ }^{2} D_{5 / 2}\right)=80.236(3) \mathrm{MHz}$ [32], respectively. The small corrections described in Sec. IV A are not included in the figures. 
TABLE VII. Values of $Q$ and $A$ for the $D_{3 / 2}$ state, calculated in configuration-interaction approach, with single and unrestricted double and triple substitutions, in several different multiconfiguration expansions; from - spectroscopic orbitals opened for substitutions; to - virtual orbital set; and $\mathrm{NCF}$ - number of configurations (see text for further details).

\begin{tabular}{cccccc}
\hline \hline & Expt. & & & \multicolumn{2}{c}{$199.8425(2)$} \\
\cline { 1 - 3 } \cline { 5 - 6 } from & to & NCF & & $\begin{array}{c}A \\
(\mathrm{MHz})\end{array}$ & $\begin{array}{c}Q \\
(\mathrm{mb})\end{array}$ \\
\hline 5 5spd6s & $1 s p d$ & 265183 & & 198.955 & 520.346 \\
$5 s p d 6 s$ & $1 s p d f$ & 386326 & & 194.391 & 533.464 \\
$5 s p d 6 s$ & $1 s p d f g$ & 641227 & & 193.744 & 536.620 \\
$5 s p d 6 s$ & $1 s p d f g h$ & 1012615 & & 193.246 & 537.786 \\
$5 s p d 6 s$ & $2 s p d 1 f$ & 943544 & & 198.752 & 522.361 \\
$5 s p d 6 s$ & $2 s p d f$ & 1543051 & & 199.973 & 520.536 \\
$5 s p d 6 s$ & $3 s p d 2 f$ & 1200261 & & 198.207 & 520.267 \\
$5 s p d 6 s$ & $3 p s d f$ & 1309130 & & 198.254 & 520.096 \\
\hline \hline
\end{tabular}

mation for both $5 d^{9} 6 s^{2}{ }^{2} D_{3 / 2}$ and $5 d^{9} 6 s^{2}{ }^{2} D_{5 / 2}$ states. In case of the $A$ values, convergence required three, rather than two, layers.

Table IX shows the effect of quadruple substitutions. The first line represents an approximation in which single, double, and triple substitutions from $5 s p d 6 s$ orbitals to a truncated virtual layer composed of " $s$," " $p$," and " $d$ " symmetries were included. The third line represents a similar approximation in which the (still truncated) virtual layer was composed of "s," " $p$," " $d$," and " $f$ " symmetries. The second, fourth, and fifth lines represent corresponding "quadruple approximation" in which single, double, triple, and quadruple substitutions were allowed. The comparison had to be made on a reasonably small orbital set in order to be able to converge the calculation involving quadruple substitutions. The numbers of CSFs in the last two lines are different because certain restrictions were applied in the calculation represented by the fourth line (see the comments near the end of Sec. III D for details). The results presented in Table IX in-

TABLE VIII. Values of $Q$ and $A$ for the $D_{5 / 2}$ state, calculated in configuration-interaction approach, with single and unrestricted double and triple substitutions, in several different multiconfiguration expansions; from-spectroscopic orbitals opened for substitutions; to-virtual orbital set; and $\mathrm{NCF}$ - number of configurations (see text for further details).

\begin{tabular}{cccccc}
\hline \hline \multicolumn{3}{c}{ Expt. } & & & \multicolumn{2}{c}{$80.236(3)$} \\
\cline { 5 - 6 } \cline { 5 - 6 } from & to & NCF & & $\begin{array}{c}A \\
(\mathrm{MHz})\end{array}$ & $\begin{array}{c}Q \\
(\mathrm{mb})\end{array}$ \\
\hline 5 5spd6s & 1 spd & 341440 & & 81.6955 & 514.929 \\
$5 s p d 6 s$ & $1 s p d f$ & 456506 & & 82.1357 & 520.259 \\
$4 f 5 s p d 6 s$ & $1 s p d f$ & 1403860 & & 79.9343 & 518.380 \\
$5 s p d 6 s$ & $1 s p d f g$ & 842883 & & 80.2371 & 519.291 \\
$5 s p d 6 s$ & $2 s p d f$ & 1326851 & 83.0623 & 521.862 \\
\hline \hline
\end{tabular}

TABLE IX. Values of $Q$ and $A$ for the $D_{3 / 2}$ state, calculated in configuration-interaction approach, with single and unrestricted double, triple, and quadruple substitutions, in several different multiconfiguration expansions; type-substitution multiplicity; fromspectroscopic orbitals opened for substitutions; to-virtual orbital set; and $\mathrm{NCF}$ - number of configurations (see text for further details).

\begin{tabular}{|c|c|c|c|c|c|}
\hline \multicolumn{4}{|c|}{ Expt. } & \multicolumn{2}{|c|}{$199.8425(2)$} \\
\hline type & from & to & $\mathrm{NCF}$ & $\begin{array}{c}A \\
(\mathrm{MHz})\end{array}$ & $\underset{(\mathrm{mb})}{Q}$ \\
\hline SDT & $5 s p d 6 s$ & $1 s p d$ & 386326 & 194.391 & 533.464 \\
\hline SDTQ & $5 s p d 6 s$ & $1 s p d$ & 569497 & 194.301 & 533.653 \\
\hline SDT & $5 s p d 6 s$ & $1 s p d f$ & 386326 & 194.391 & 533.464 \\
\hline SDTQ & $5 s p d 6 s$ & $1 s p d f$ & 967871 & 195.376 & 531.685 \\
\hline SDTQ & $5 s p d 6 s$ & $1 s p d f$ & 1089014 & 194.686 & 531.846 \\
\hline
\end{tabular}

dicate that the correction involving quadruple substitutions is unlikely to exceed $1 \%$. The CSFs representing quadruple substitutions were not carried over to the following calculations and the "quadruple" correction was included additively, as described in Sec. IV A.

\section{Four-dimensional configuration-interaction calculations}

A full converged CAS calculation for the gold atom is still unattainable due to software and hardware limitations. Based on our current calculations we estimate that the CAS approach would require configuration expansions in four dimensions: (1) single, double, triple, and perhaps quadruple substitutions, (2) from all core shells (or at least from $3 s p d 4 s p d f 5 s p d 6 s)$, (3) to eight or more virtual orbital layers, (4) of " $s, "$ " $p$, , " $d$, , " $f$," " $g$," " $h$," and perhaps higher symmetries. One can imagine a "space" spanned by the four "dimensions" defined above, i.e., substitution multiplicity, number of opened core subshells, number of virtual layers, and maximal symmetry of virtual layer dimension. In fact, this space should rather be called a "matrix" since all four dimensions are discrete. Let us call this four-dimensional matrix a "CAS matrix." Each element of the matrix is represented by a multiconfiguration expansion obtained by substituting a particular number of electrons (substitution dimension) from specific core orbitals (core dimension) to a set of virtual orbitals (virtual dimension) of specific symmetries (symmetry dimension). A full CAS calculation would require several orders of magnitude larger configuration expansions than are possible even with the largest computer resources available today.

However, a computational strategy can be designed in which a considerably smaller multiconfiguration expansion yields a wave function only marginally inferior to a full CAS wave function in the sense that all important electron correlation effects are included and the calculated values of $A$ and $Q$ are close to those that would result from a full, converged, CAS calculation. The strategy is based on the observation that one does not have to simultaneously push the configuration expansions to the limits of all the above mentioned 
dimensions. Specifically, the dependence of atomic properties on the substitution dimension is critical. To illustrate this approach, let us consider separately the contributions of single, double, and triple substitutions to the calculated values of $A$ and $Q$ of gold. To obtain converged results within a single substitution model, one has to include substitutions from all occupied shells ( $1 s 2 s p 3 s p d 4 s p d f 5 s p d 6 s)$ to eight or more virtual layers. This is illustrated in Tables I-IV, where eight virtual orbital layers were necessary to converge the series of self-consistent-field calculations. However, to obtain a converged result within a single and double substitution model (SD) one has to include double substitutions from $4 s p d f 5 s p d 6 s$ occupied orbitals, not to eight but to three or at most four virtual layers (see Tables V and VI). In the single, double, and triple substitution model (SDT) it is enough to consider triple substitutions from $5 \operatorname{spd} 6 s$ occupied orbitals to two or at most three virtual layers (see Tables VII and VIII). In the space (or rather in the matrix) of the four dimensions defined above, the core and virtual dimension sizes strongly depend on the substitution dimension (in fact, all four dimensions are interdependent).

Therefore, one can construct an approximation, in which all important electron correlation effects are included and the calculated values of $A$ and $Q$ are close to those that would result from a full converged CAS calculation. In order to find a suitable approximation, we have performed a set of test calculations for several elements of the above mentioned matrix. For each dimension, the calculations were saturated to the point where the relative change in the expectation values (i.e., both $A$ and $Q$ ) did not exceed a small fraction of a percent (usually two or three tenths of a percent). Specifically, for each substitution dimension (i.e., for single, double, and triple substitutions) we thoroughly tested the dependence of observables on symmetry, virtual, and core spaces. When a saturated set of CSFs is obtained for a particular substitution dimension, all these CSFs are carried over to the next step(s). The merged final multiconfiguration expansion represents an approximation, which is effectively equivalent to a CAS expansion, and the corresponding wave function is of similar quality as a CAS wave function, at least from the point of view of the calculated values of $A$ and $Q$.

In practice there is not one single final "CAS" expansion, but a series of such final expansions in which various sets of "S," "SD," and "SDT" multiconfiguration expansions (i.e., various sets with single, double, and triple substitutions) are merged together. Table $\mathrm{X}$ shows the results obtained from a series of such final CAS calculations for the ${ }^{2} D_{3 / 2}$ state, and Table XI shows the same for the ${ }^{2} D_{5 / 2}$ state. The data from both tables are also included in Fig. 3. The CAS expansions are composed as follows. All virtual orbitals and all CSFs generated in the $\mathrm{SrD}$ approximation, as described in Sec. III A, as well as those described in Sec. III B, were included. The remaining CSF expansions were generated with substitutions from $5 s p d 6 s$ orbitals to virtual sets described in the first column of Tables X and XI, where symbols before the colon represent substitution multiplicity-i.e., SD for single and double substitutions while SDT for single and double and triple substitutions - and symbols after the colon represent virtual orbital layers-i.e., 3 hgg for three layers (first layer with "spdfgh" symmetries and two layers with "spdfg"
TABLE X. The final configuration-interaction calculations of $Q$ and $A$ for the $5 d^{9} 6 s^{2}{ }^{2} D_{3 / 2}$ state of $\mathrm{Au}$; type-description of the multiconfiguration expansions (see text for details); $\mathrm{NCF}=$ size of the multiconfiguration expansion.

\begin{tabular}{cccccc}
\hline \hline \multicolumn{2}{c}{ Expt. } & & & \multicolumn{2}{c}{$199.8425(2)$} \\
\cline { 5 - 6 } \cline { 5 - 6 } type & NCF & & $\begin{array}{c}A \\
(\mathrm{MHz})\end{array}$ & $\begin{array}{c}Q \\
(\mathrm{mb})\end{array}$ \\
\hline SD:3hgg+SDT:2fd & 1182329 & & 206.343 & 517.201 \\
SD:3hgf+SDT:2fd & 1144532 & & 206.221 & 517.342 \\
SD:3hgf+SDT:2gd & 1711382 & & 205.104 & 519.106 \\
SD:3hgf+SDT:2gf & 1847380 & & 204.489 & 519.829 \\
\hline \hline
\end{tabular}

symmetries); $2 f d$ for two layers (first layer with "spdf" symmetries and second layer with "spd" symmetries); $3 h g f$ for three layers (first layer with "spdfgh" symmetries, second layer with "spdfg" symmetries, and third layer with "spdf" symmetries), etc.

In the largest calculations, when single, double, and triple substitutions to two or three layers were included, we had to further limit the overall number of CSFs due to software and hardware limitations. In those cases, the occupation number of the least important virtual orbital was restricted to single or double, thus excluding those CSFs in which this particular virtual orbital was occupied by three electrons. The difference that such a restriction brings about can always be evaluated on a smaller set of CSFs before a full calculation is performed. Therefore we always had control on the effects of the above mentioned restrictions on the calculated values of $A$ and $Q$.

\section{RESULTS}

More extensive calculations turned out to be beyond the 100 node limit for this project on the Linux cluster at the National Institute of Standards and Technology (NIST), USA. Therefore the calculations of the magnetic dipole constants $A$ did not yield converged results. As might be expected, the effects of double and triple substitutions are relatively larger for $A$ than for $Q$, therefore the calculations of the $Q$ values were essentially converged; they yield $Q\left({ }^{2} D_{3 / 2}\right)=519.829 \mathrm{mb}$ and $Q\left({ }^{2} D_{5 / 2}\right)=522.066 \mathrm{mb}$, respectively.

TABLE XI. The final configuration-interaction calculations of $Q$ and $A$ for the $5 d^{9} 6 s^{2}{ }^{2} D_{5 / 2}$ state of $\mathrm{Au}$; type-description of the multiconfiguration expansions (see text for details); $\mathrm{NCF}=$ size of the multiconfiguration expansion.

\begin{tabular}{cccccc}
\hline \hline \multicolumn{2}{c}{ Expt. } & & & \multicolumn{2}{c}{$80.236(3)$} \\
\cline { 1 - 3 } \cline { 5 - 6 } type & NCF & & $\begin{array}{c}A \\
(\mathrm{MHz})\end{array}$ & $\begin{array}{c}Q \\
(\mathrm{mb})\end{array}$ \\
\hline SD:3hgf+SDT:2fd & 1441120 & & 78.2451 & 520.073 \\
SD:3hgf+SDT:2gd & 1527668 & & 79.9182 & 522.066 \\
\hline \hline
\end{tabular}




\section{A. Corrections}

As mentioned in Sec. III C, the contributions arising from unrestricted double substitutions from $4 s p d f$ orbitals were evaluated separately and included additively in the final $Q$ values. They yield +0.312 and $+1.498 \mathrm{mb}$ for the two states ${ }^{2} D_{3 / 2}$ and ${ }^{2} D_{5 / 2}$, respectively. The effects of the quadruple substitutions were also evaluated separately, in a very limited fashion, and only for the ${ }^{2} D_{3 / 2}$ state. As explained in Sec. III $\mathrm{C}$, the correction arising from the quadruple substitutions for the ${ }^{2} D_{3 / 2}$ state lowers the $Q$ value by $1.779 \mathrm{mb}$. The dependence of the $Q$ values on double and triple substitutions indicates that the quadruple correction might be smaller for the $Q\left({ }^{2} D_{5 / 2}\right)$ value than for the $Q\left({ }^{2} D_{3 / 2}\right)$ value, but we were unable to evaluate the former. Therefore we assumed identical, $-1.8 \mathrm{mb}$, corrections for both states. The corrections arising from the Breit interaction were calculated at the Dirac-Hartree-Fock level with full relaxation, i.e., with a frequency-dependent Breit term

$$
\begin{aligned}
B_{i j}= & -\frac{\boldsymbol{\alpha}_{i} \cdot \boldsymbol{\alpha}_{j}}{r_{i j}}-\frac{\boldsymbol{\alpha}_{i} \cdot \boldsymbol{\alpha}_{j}}{r_{i j}}\left[\cos \left(\omega_{i j} r_{i j}\right)-1\right] \\
& +c^{2}\left(\boldsymbol{\alpha}_{i} \cdot \vec{\nabla}_{i}\right)\left(\boldsymbol{\alpha}_{j} \cdot \vec{\nabla}_{j}\right) \frac{\cos \left(\omega_{i j} r_{i j} / c\right)-1}{\omega_{i j}^{2} r_{i j}}
\end{aligned}
$$

included in the self-consistent-field functional, using the MCDFGME code [2,4,39]. In the formula above, $r_{i j}=\left|\vec{r}_{i}-\vec{r}_{j}\right|$ is the interelectronic distance, $\omega_{i j}$ is the energy of the photon exchanged between two electrons, $\boldsymbol{\alpha}_{i}$ are Dirac matrices, and $c=1 / \alpha$ is the speed of light. The Breit corrections are highly state dependent (see also [19], where the Gaunt part was evaluated) and yield 2.3 and $0.6 \mathrm{mb}$ for the two states, ${ }^{2} D_{3 / 2}$ and ${ }^{2} D_{5 / 2}$, respectively. The quantum electrodynamics (QED) corrections to the $Q$ values are expected to be very small. We evaluated the vacuum polarization (VP) correction with the MCDFGME code, following Ref. [40], and obtained a value on the order of $0.01 \%$. When all above mentioned corrections are included, the $Q$ values become $Q\left({ }^{2} D_{3 / 2}\right)=520.641 \mathrm{mb}$ and $Q\left({ }^{2} D_{5 / 2}\right)=522.364 \mathrm{mb}$. The average of the above two results yields $Q\left({ }^{197} \mathrm{Au}\right)=521.5 \mathrm{mb}$.

\section{B. Error estimate}

A rigorous systematic treatment of the error bar of the calculated electric quadrupole moment $Q$ would require evaluation of the effects of all omitted virtual orbitals, all CSFs which were not included in the configuration expansions, as well as all physical effects that were not included or were treated approximately. However, we were only able to obtain very crude estimates of certain sources of systematic errors. We believe that none exceeded $1 \%$, but the calculations presented in this paper were far too extensive to permit a rigorous treatment of the error. Therefore we have to resort to a less rigorous method.

One of the frequently used methods of evaluation of the accuracy of calculated electric quadrupole moments $Q$ is based on the simultaneous calculations of magnetic dipole hyperfine constants $A$ and on subsequent comparison of calculated $A$ values with their experimental counterparts. As mentioned above, the calculations of the magnetic dipole

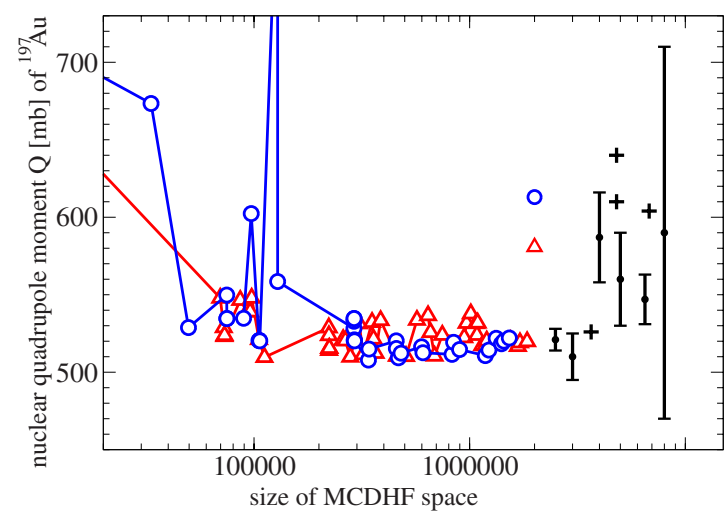

FIG. 4. (Color online) Nuclear quadrupole moment $Q(\mathrm{mb})$ of the ${ }^{197} \mathrm{Au}$ isotope obtained from the calculated electric field gradients as a function of the size of multiconfiguration expansions for the states $5 d^{9} 6 s^{2}{ }^{2} D_{3 / 2}$ (triangles-red online) and $5 d^{9} 6 s^{2}{ }^{2} D_{5 / 2}$ (circles-blue online), compared with other theoretical and experimental results. The small corrections described in Sec. IV A are not included in the figure. The values which represent multiconfiguration expansions of sizes smaller than 20000 are outside the figure, except the uncorrelated Dirac-Hartree-Fock values, represented by the single triangle (red online) for ${ }^{2} D_{3 / 2}$ and the single circle (blue online) for ${ }^{2} D_{5 / 2}$. The six values with error bars are from Refs. [18-20,32,37,38]; the four values without error bars, represented by pluses, are from Refs. [17,21,31]; all data are arranged in reverse chronological order, with the most recent results to the left.

constants $A$ have not converged. However, the amplitudes of the final oscillations of the two curves representing the values of $A$ for the two states of interest are comparable to the uncertainty of $A$ arising from the accuracy of the nuclear magnetic dipole moment value $\mu$.

There are currently two different $\mu$ values in the literature $[28,29], \mu=0.145746(9)$ and $\mu=0.148$ 158(8), which differ by about $2 \%$. Taken at face value, our results seem to favor the smaller value, $\mu=0.145746(9)$, which, as mentioned in Sec. II, has been used in the present calculations. However, the overall accuracy of our calculations (in particular, the evaluation of higher-order terms) does not permit us to draw a definitive conclusion. Therefore, the difference between the two values of $\mu$ should rather be treated as a source of systematic error in the determination of $A$. Therefore, we did not push the calculations of magnetic dipole constants $A$ further beyond their current level of convergence and, consequently, the calculations of $A$ values could not be used as reliable sources of error estimate for nuclear moments.

Another method to estimate the accuracy of $Q$ is to consider the differences between the final values obtained from different states. However, in the present paper we were able to converge the calculations for only two atomic levels. The difference between the results obtained for these two levels turned out to be quite small, which rendered this method useless in this particular case.

Considering the computational methodology employed in this paper, it is obvious that the final value depends on the choice of the multiconfiguration expansions representing the last few points on the curves in Fig. 4, while the accuracy of the final value is connected with convergence of these 
TABLE XII. Comparison of the present $Q\left({ }^{197} \mathrm{Au}\right)$ value (in $\mathrm{mb}=10^{-31} \mathrm{~m}^{2}$ ) with other recent values and with previous (muonic) standard value.

\begin{tabular}{cll}
\hline \hline Ref. & \multicolumn{1}{c}{ Source } & $Q\left({ }^{197} \mathrm{Au}\right)$ \\
\hline This work & Au atom $,{ }^{2} D_{3 / 2},{ }^{2} D_{5 / 2}$ & $521.5 \pm 5.0$ \\
{$[19]$} & Au atom, ${ }^{2} D_{3 / 2},{ }^{2} D_{5 / 2}$ & $521 \pm 7$ \\
{$[20]$} & AuF, LAuF molecules & $510 \pm 15$ \\
{$[21]$} & $\mathrm{AuX}, \mathrm{LAu} X$ molecules & 526 \\
{$[38]$} & Muonic & $547 \pm 16$ \\
\hline \hline
\end{tabular}

curves. Therefore, we based the estimate of the error bar on the oscillations of the tail of the two curves in Fig. 4. The largest differences taken from the last few points on the curves representing ${ }^{2} D_{3 / 2}$ and ${ }^{2} D_{5 / 2}$ states amount to 3 and 4 $\mathrm{mb}$, respectively. As an additional source of uncertainty we assumed the additive corrections described in Sec. IV A since all of them were evaluated in a rather crude approximation. For instance, the contribution of the Breit interaction was calculated at the Dirac-Hartree-Fock level, without regard to electron correlation effects. When all above sources of uncertainly are taken into account the total error bar amounts to $5 \mathrm{mb}$, which yields our final calculated value of quadrupole moment $Q\left({ }^{197} \mathrm{Au}\right)=521.5 \pm 5.0 \mathrm{mb}$.

\section{COMPARISONS}

The results of our calculation are compared with previous evaluations in Table XII and in Fig. 4. It is worth noting that our result is in agreement with three most recent theoretical values, obtained with three different methods, but all these recent results (including ours) are considerably smaller than other, earlier values.

Yakobi et al. [19] performed calculations for the $5 d^{9} 6 s^{2}{ }^{2} D_{3 / 2}$ and $5 d^{9} 6 s^{2}{ }^{2} D_{5 / 2}$ states of atomic gold within the four-component Dirac-Coulomb framework [41,42]. They correlated 51 out of the 79 electrons in the large basis sets (up to $26 s 22 p 18 d 12 f 8 g 5 h$ uncontracted Gaussian functions) with the relativistic Fock-space coupled-cluster (CC) method including single and double excitations (CCSD). The contribution of the Gaunt term, the main part of the Breit interaction, was also evaluated.

Belpassi et al. [20] performed molecular relativistic Dirac-Coulomb-Gaunt Hartree-Fock calculations [43] for a series of molecules: AuF, XeAuF, KrAuF, ArAuF, (OC)AuF, and $\mathrm{AuH}$. The electronic correlation contributions were included at $\operatorname{CCSD}(\mathrm{T})$ and CCSD-T levels. The value of the nuclear quadrupole moment $Q$ was obtained from the determinations of the electric field gradient at the gold nucleus for the above mentioned molecules, combined with experimental values of the nuclear quadrupole coupling constants.

Thierfelder et al. [21] performed four-component relativistic density-functional theory (DFT) calculations for diatomic compounds $\mathrm{Cu} X$ and $\mathrm{Au} X(X=\mathrm{H}, \mathrm{F}, \mathrm{Cl}, \mathrm{Br}$, and $\mathrm{I})$ with and without $\mathrm{CO}$ attached, i.e., $\mathrm{OC}-\mathrm{Cu} X$ and $\mathrm{OC}-\mathrm{Au} X$ $(X=\mathrm{F}, \mathrm{Cl}, \mathrm{Br}$, and $\mathrm{I})$. They employed a newly developed functional [44], whose role is to correctly describe the long- range part of exchange interactions [45], and obtained the averaged result $Q=526 \mathrm{mb}$. This value is within the error bounds of our value.

Our result, in turn, falls within the error bounds published by Belpassi et al. [20] $[Q=510(15) \mathrm{mb}$, as well as those by Yakobi et al. [19] [Q=521(7) mb]. The agreement with Yakobi et al. [19] may be somewhat accidental because particular contributions show larger differences. The two outstanding differences arise from triple substitutions and from deep core orbitals. Yakobi et al. [19] evaluated the effect of the triple substitutions by performing single-reference $\operatorname{CCSD}(\mathrm{T})$ calculation for the ${ }^{2} D_{5 / 2}$ level and obtained a $0.3 \%$ shift. The effect of triple substitutions is indeed smaller for the ${ }^{2} D_{5 / 2}$ level, but for the ${ }^{2} D_{3 / 2}$ level our calculations indicate a shift on the order of $2 \%$. However, this discrepancy may be attributed to the methodological differences in the two papers. The definition of triple substitutions in the configurationinteraction method used here differs substantially from that in the $\operatorname{CCSD}(\mathrm{T})$ approach due to the exponential nature of the coupled-cluster operator. The coupled-cluster approximation includes a subset of the CI triple substitutions (the "unlinked" diagrams), as well as that of higher-order substitutions, already at the CCSD level. The $\operatorname{CCSD}(\mathrm{T})$ yields only the "linked" part as the effect of the triple substitutions. Therefore, the contribution of the CI triple substitutions may indeed be expected to be larger than that of the $\mathrm{CC}$ triple substitutions.

Another difference arises from contributions of deep core orbitals. The effects of $3 s p d, 2 s p$, and $1 s$ orbitals were neglected by Yakobi et al. [19], while in our calculations they were all included. Their combined effect was to lower the $Q$ value by about $2 \%$.

\section{CONCLUSIONS}

The multiconfiguration Dirac-Hartree-Fock (MCDHF) model has been employed to calculate the expectation values responsible for the hyperfine splittings of the $5 d^{9} 6 s^{2}{ }^{2} D_{3 / 2}$ and $5 d^{9} 6 s^{2}{ }^{2} D_{5 / 2}$ levels of atomic gold. All one-, two-, and three-body electron correlation effects were included and saturated for electric quadrupole hyperfine values of a heavy, open-shell, neutral atom. The correlation effects involving all 79 electrons were accounted for with a procedure that is equivalent to a full complete-active-space calculation. All electron correlation effects were explicitly accounted for at a $1 \%$ level of accuracy or better. Calculated electric field gradients, together with experimental values of the electric quadrupole hyperfine-structure constants $B$, allow us to extract a nuclear electric quadrupole moment $Q$ $=521.5(5.0) \mathrm{mb}$ of ${ }^{197} \mathrm{Au}$. If taken at face value, the summary in Table XII suggests that our $Q$ value, together with that of Yakobi et al. [19], could become the standard value.

\section{ACKNOWLEDGMENTS}

This work was supported by the Polish Ministry of Science and Higher Education (MNiSW) in the framework of scientific Grant No. 1 P03B 11030 available for the years 2006-2009. P.J. acknowledges support from the Swedish 
Research Council (Vetenskapsrådet). P.P. belongs to the Finnish Center of Excellence in Computational Molecular Science (CMS). The visits of J.B. at Helsinki were supported by The Academy of Finland. The large scale calculations were performed on the Raritan Linux cluster at the National Institute of Standards and Technology (NIST) in Gaithersburg, USA. J.B. would like to express his gratitude for the hospitality which was extended to him during his visits to the Chemistry Department of the University of
Helsinki and the Atomic Spectroscopy Group at NIST. P.I. acknowledges the support of the Helmholtz Alliance Program of the Helmholtz Association under Contract No. HA216 ("Extremes of Density and Temperature: Cosmic Matter in the Laboratory"). Laboratoire Kastler Brossel is "Unité Mixte de Recherche du CNRS, de l'ENS et de l'UPMC No. 8552." We thank the (anonymous) referee for pointing our attention to the structural differences between $\mathrm{CI}$ and $\mathrm{CC}$ methods.
[1] P. Jönsson, X. He, C. Froese Fischer, and I. P. Grant, Comput. Phys. Commun. 177, 597 (2007).

[2] P. Indelicato and J.-P. Desclaux, MCDFGME, A Multiconfiguration Dirac-Fock and General Matrix Element Program, 2005 (http://dirac.spectro.jussieu.fr/mcdf).

[3] J.-P. Desclaux, Comput. Phys. Commun. 9, 31 (1975).

[4] J.-P. Desclaux, in Methods and Techniques in Computational Chemistry, edited by E. Clementi (STEF, Cagliary, 1993), Vol. A, p. 253.

[5] I. P. Grant, Relativistic Quantum Theory of Atoms and Molecules: Theory and Computation (Springer, New York, 2007).

[6] I. Lindgren and J. Morrison, Atomic Many-Body Theory (Springer-Verlag, New York, 1986).

[7] V. A. Dzuba, Phys. Rev. A 71, 032512 (2005).

[8] V. A. Dzuba and V. V. Flambaum, Phys. Rev. A 75, 052504 (2007).

[9] W. R. Johnson, Atomic Structure Theory: Lectures on Atomic Physics (Springer, Berlin, 2007).

[10] J. Bieroń, P. Jönsson, and C. Froese Fischer, Phys. Rev. A 53, 2181 (1996).

[11] J. Bieroń, P. Jönsson, and C. Froese Fischer, Phys. Rev. A 60, 3547 (1999).

[12] V. A. Yerokhin, Phys. Rev. A 77, 020501(R) (2008).

[13] J. Autschbach, S. Siekierski, M. Seth, P. Schwerdtfeger, and W. H. E. Schwarz, J. Comput. Chem. 23, 804 (2002).

[14] I. Malkin, O. L. Malkina, V. G. Malkin, and M. Kaupp, Chem. Phys. Lett. 396, 268 (2004).

[15] E. Malkin, I. Malkin, O. L. Malkina, V. G. Malkin, and M. Kaupp, Phys. Chem. Chem. Phys. 8, 4079 (2006).

[16] S. Q. Song, G. F. Wang, A. P. Ye, and G. Jiang, J. Phys. B 40, 475 (2007).

[17] P. Schwerdtfeger et al., J. Chem. Phys. 122, 124317 (2005).

[18] W. M. Itano, Phys. Rev. A 73, 022510 (2006).

[19] H. Yakobi, E. Eliav, and U. Kaldor, J. Chem. Phys. 126, 184305 (2007).

[20] L. Belpassi, F. Tarantelli, A. Sgamellotti, H. M. Quiney, J. N. P. van Stralen, and L. Visscher, J. Chem. Phys. 126, 064314 (2007).

[21] C. Thierfelder, P. Schwerdtfeger, and T. Saue, Phys. Rev. A
76, 034502 (2007).

[22] P. Pyykkö, Angew. Chem., Int. Ed. 43, 4412 (2004) (German); Angew. Chem. 116, 4512 (2004).

[23] P. Pyykkö, Inorg. Chim. Acta 358, 4113 (2005).

[24] P. Pyykkö, Chem. Soc. Rev. 37, 1967 (2008).

[25] P. Pyykkö, Mol. Phys. 106, 1965 (2008).

[26] I. P. Grant, Comput. Phys. Commun. 84, 59 (1994).

[27] K. G. Dyall, I. P. Grant, C. T. Johnson, F. A. Parpia, and E. P. Plummer, Comput. Phys. Commun. 55, 425 (1989).

[28] P. Raghavan, At. Data Nucl. Data Tables 42, 189 (1989).

[29] N. J. Stone, At. Data Nucl. Data Tables 90, 75 (2005).

[30] P. Jönsson, F. A. Parpia, and C. Froese Fischer, Comput. Phys. Commun. 96, 301 (1996).

[31] A. G. Blachman, D. A. Landman, and A. Lurio, Phys. Rev. 161, 60 (1967).

[32] W. J. Childs and L. S. Goodman, Phys. Rev. 141, 176 (1966).

[33] J. Bieroń, C. Froese Fischer, P. Jönsson, and P. Pyykkö, J. Phys. B 41, 115002 (2008).

[34] P. Pyykkö, Chem. Rev. (Washington, D.C.) 88, 563 (1988).

[35] P. Jönsson, A. Ynnerman, C. Froese Fischer, M. R. Godefroid, and J. Olsen, Phys. Rev. A 53, 4021 (1996).

[36] M. Godefroid, G. V. Meulebeke, P. Jönsson, and C. Froese Fischer, Z. Phys. D: At., Mol. Clusters 42, 193 (1997).

[37] P. Palade, F. E. Wagner, A. D. Jianu, and G. Filoti, J. Alloys Compd. 353, 23 (2003).

[38] R. J. Powers, P. Martin, G. H. Miller, R. E. Welsh, and D. A. Jenkins, Nucl. Phys. A 230, 413 (1974).

[39] P. Indelicato, Phys. Rev. A 51, 1132 (1995).

[40] S. Boucard and P. Indelicato, Eur. Phys. J. D 8, 59 (2000).

[41] E. Eliav, U. Kaldor, and Y. Ishikawa, Phys. Rev. A 49, 1724 (1994).

[42] U. Kaldor and E. Eliav, Adv. Quantum Chem. 31, 313 (1998).

[43] M. Pernpointner and L. Visscher, J. Chem. Phys. 114, 10389 (2001).

[44] T. Yanai, D. P. Tew, and N. C. Handy, Chem. Phys. Lett. 393, 51 (2004).

[45] E. Goll, H. Stoll, C. Thierfelder, and P. Schwerdtfeger, Phys. Rev. A 76, 032507 (2007). 